\title{
Fine-Scale Spatial Organization of Face and Object Selectivity in the Temporal Lobe: Do Functional Magnetic Resonance Imaging, Optical Imaging, and Electrophysiology Agree?
}

\author{
Hans P. Op de Beeck, ${ }^{1}$ James J. DiCarlo, ${ }^{2 *}$ Jozien B. M. Goense, ${ }^{3 *}$ Kalanit Grill-Spector, ${ }^{4 *}$ Alex Papanastassiou, ${ }^{2 *}$ \\ Manabu Tanifuji, ${ }^{5 *}$ and Doris Y. Tsao ${ }^{6,7 *}$ \\ ${ }^{1}$ Laboratory of Experimental Psychology, University of Leuven (Katholieke Universiteit Leuven), B-3000 Leuven, Belgium, ${ }^{2}$ McGovern Institute for Brain \\ Research, Massachusetts Institute of Technology, Cambridge, Massachusetts 02139, ${ }^{3}$ Max Planck Institute for Biological Cybernetics, 72076 Tuebingen, \\ Germany, ${ }^{4}$ Department of Psychology and Neurosciences Institute, Stanford University, Stanford, California 94305, ${ }^{5}$ Laboratory Integrative Neural Systems, \\ Riken Brain Science Institute, Saitama 351-0198, Japan, ${ }^{6}$ Institute for Brain Research, D-28359 Bremen, Germany, and ${ }^{7}$ Division of Biology, California \\ Institute of Technology, Pasadena, California 91125
}

The spatial organization of the brain's object and face representations in the temporal lobe is critical for understanding high-level vision and cognition but is poorly understood. Recently, exciting progress has been made using advanced imaging and physiology methods in humans and nonhuman primates, and the combination of such methods may be particularly powerful. Studies applying these methods help us to understand how neuronal activity, optical imaging, and functional magnetic resonance imaging signals are related within the temporal lobe, and to uncover the fine-grained and large-scale spatial organization of object and face representations in the primate brain.

Key words: face perception; object recognition; single unit; local field potential; optical imaging; fMRI

Primates have a great capacity to categorize and identify faces and other objects. No artificial intelligent device has ever been created with the same object recognition capabilities as the human brain, despite the speed of modern-day computers. The details of how the primate brain accomplishes this task are still not well known, but we know where to look: the temporal lobe of the brain. Interest in the functional properties of regions in the temporal lobe has been increasing ever since early primate lesion studies showed its importance for learning and object recognition (Dean, 1976). Regretfully, the location of the temporal lobe at the ventral side of the brain makes it cumbersome to access with invasive techniques. In addition, the study of temporal lobe neurons is challenging because these neurons often respond only to a small subset of stimuli. Indeed, most researchers studying the temporal lobe in monkeys have experienced the frustration of investing weeks of time inserting electrodes in this part of the brain before finally finding some recording positions where neurons respond strongly to the visual images included in the experiment. So the importance of understanding functional organization in the temporal lobe is abundantly clear from a practical point of view, but

Received Aug. 11, 2008; accepted Sept. 10, 2008.

This work was supported by Human Frontier Science Program Grant CDA-0040/2008 (H.P.0.d.B.), the Fund for Scientific Research-Flanders (H.P.O.d.B.), The Pew Charitable Trusts (UCSF 2893sc) (J.J.D.), The Max Planck Society (J.B.M.G.), Whitehall Foundation Grant 2005-05-111-RES (K.G.-S.), and The Humboldt Foundation (D.Y.T).

*J.J.D., J.B.M.G., K.G.-S., A.P., M.T., and D.Y.T. contributed equally to this work.

Correspondence should be addressed to Hans P. Op de Beeck, Laboratory of Experimental Psychology, Katholieke Universiteit Leuven, Tiensestraat 102, B-3000 Leuven, Belgium. E-mail: hans.opdebeeck@psy.kuleuven.be. DOI:10.1523/JNEUROSCI.3799-08.2008

Copyright $\odot 2008$ Society for Neuroscience ～0270-6474/08/2811796-06\$15.00/0 most importantly, it would help us to understand the neural mechanisms behind the superior recognition performance at the behavioral level.

Here, we will focus on several techniques, invasive and noninvasive, and their application in the temporal lobe. To address questions of brain organization, we have several methods at our disposal, including electrophysiology, optical imaging, and functional magnetic resonance imaging (fMRI). In humans, mostly the noninvasive methods are relevant. Two parameters are important for assessing the usefulness of each technique: spatial resolution and coverage. Electrophysiological, extracellular recordings provide single-neuron spatial resolution but focus on a small, potentially unrepresentative local sample of neurons, and optical imaging provides tens of micrometer resolution and a coverage of a few millimeters. For fMRI, the spatial resolution depends on coverage. The spatial resolution is typically a few millimeters for whole-brain studies, but for localized fMRI or in small animals resolution can be $\sim 200 \mu \mathrm{m}$ or less (Fukuda et al., 2006; Harel et al., 2006; Goense et al., 2007).

The first studies investigating the relationships between these techniques have focused on primary visual cortex (V1). The function and organization of this region is so well known from electrophysiology that this area can be used as a test bed for validating new techniques. V1 data showed early on how columns defined by optical imaging relate to extracellular spiking activity (Grinvald et al., 1986). Likewise, orientation columns identified through contrast-enhanced fMRI correspond to orientation columns visualized with optical imaging (Fukuda et al., 2006). Fur- 
thermore, V1 data demonstrated that the fMRI signal obtained from the intrinsic blood oxygenation level-dependent (BOLD) contrast is slightly better correlated with an indirect measure of synaptic activity [local field potentials (LFPs)] than with multiunit spiking activity (MUA) (Logothetis et al., 2001).

Here, we are not so much interested in the relationships between these techniques per se, but in how the combination of these techniques can help us to clarify the functional organization of regions in the temporal lobe. Our understanding of this functional organization is growing steadily, but is still not sufficient to address the big question of how the spatial organization of the temporal lobe relates to its functional role in visual object recognition. One hypothesis is that there are domain-specific systems containing neurons and circuitry for classes of complex objects such as faces, bodies, etc. Another hypothesis is that there is alphabet of small columns where neurons inside each column prefer a particular feature and the active combination of columns represents each object. A third possibility is that neurons that participate in representing the same object are fully intermixed, perhaps organized into multiple interleaved feature maps.

Here, we will focus on how the combination of electrophysiological and imaging techniques can help unravel the functional organization of the temporal lobe. We will first briefly describe what each technique in isolation has revealed and then we will illustrate the power of their combination. In comparing across techniques, we will address two central questions. First, what are the processes that contribute to the signal measured with each of these methods? Although positive correlations have been found between the different methods, these correlations are smaller than expected given the reliability of the data. This opens the possibility that different methods measure different signals, for example, a differently weighted contribution of the input and the output of neurons. Second, what is the degree and spatial scale of clustering of neurons with similar properties in the temporal lobe? The answers from different techniques (e.g., optical imaging vs fMRI) and different designs (e.g., use of different stimuli) are qualitatively similar, but quantitatively different.

\section{Electrophysiology in monkeys}

Gross et al. (1969, 1972) gave an early, qualitative description of neural receptive fields and response properties of neurons in primate inferior temporal (IT) cortex, suggesting cells with relatively large receptive fields that are selective for complex visual stimuli like faces and hands. Decades later, these findings are still valid, although significant research has quantified and qualified these response properties of IT neurons. Many IT neurons are activated strongly by relatively complex stimuli (Perrett et al., 1982; Desimone et al., 1984), but these stimuli are often moderately complex visual features or object fragments rather than images of whole objects (Kobatake and Tanaka, 1994). Furthermore, although this stimulus selectivity is largely tolerant to image transformations like changes in position and size (Ito et al., 1995; Wallis and Rolls, 1997), single neurons nevertheless retain a surprising degree of sensitivity for such transformations (Op de Beeck and Vogels, 2000; DiCarlo and Maunsell, 2003). Computational work and population analyses of neuronal selectivity suggest that a representation of moderately complex features that is largely tolerant for metric transformations like size and position may be an effective population code allowing for invariant object recognition (Hung et al., 2005; DiCarlo and Cox, 2007). Absolute invariance and selectivity for whole objects are not necessary.
The functional organization of IT cortex is another question that has attracted much interest. Are there feature, object, or category columns/maps in IT cortex analogous to orientation columns in V1 or motion-direction maps in MT? Early singleunit electrophysiological studies suggested some clustering (Desimone and Gross, 1979; Gochin et al., 1991), but nevertheless the similarity in response properties between nearby neurons was not very high. Electrophysiology studies have also investigated regional variations in stimulus preference on a larger scale. Most notably, Baylis et al. (1987) described some regional variation in the preference for simple visual stimuli, more complex visual stimuli, and faces, but this variation was relatively modest. Fujita et al. (1992) showed the existence of a finer columnar organization with respect to the simplest visual stimuli that activate neurons efficiently (the "critical features"). A critical feature for a neuron was determined by simplifying the effective stimulus little by little without changing evoked responses of the neuron (the reduction technique). The resulting moderately complex features were similar across nearby neurons and neurons with similar preferences seemed to be organized in a columnar manner in IT cortex. The connectivity within IT cortex (between areas TEO and TE) and afferent input to IT cortex also suggests a columnar organization at a size of a few hundred micrometers (Saleem et al., 1993; Tanigawa et al., 2005).

One question is why the reduction technique proved so useful for finding clustering of neurons with similar properties, while at the same time it is not uncommon to find nearby neurons with a totally different selectivity. One possibility is that the reduction technique partially removes the effects of the intrinsic circuit (e.g., interneurons and horizontal connections) that determines how an IT neuron responds to the afferent input, and so the effect of the afferent input would be greater using the reduction technique than when using more complex stimuli. From this respect, the reduction technique might inform us what the common input is of the neurons in an IT column. Two other methods have also been successful in reducing the variability between IT neurons. First, recent studies have focused on LFPs, which might be related more to synaptic activity than to spiking output. Second, measurements of multiunit activity (instead of single-unit activity) average out the variability across neurons in the local neuronal population. The latter two methods have recently provided evidence for clustering over a distance $<500 \mu \mathrm{m}$ for multiunit activity and over a distance of $>1 \mathrm{~mm}$ for LFPs (Kreiman et al., 2006). Thus, there appear to be multiple scales and strengths of organization depending on the methodology used and signal that is measured.

\section{Optical imaging in monkeys}

Activation in neural tissues elicits changes in optical properties of the tissue. Optical imaging, more specifically optical intrinsic signal imaging (OISI), is a technique to visualize these changes (intrinsic signals) from an exposed cortical surface with CCD cameras. Typically, a wavelength of light $\sim 610 \mathrm{~nm}$ is used for measurements of the intrinsic signals in IT cortex. The major source of the changes at this wavelength is the absorption changes reflecting increase of deoxyhemoglobin in capillaries due to increased neural activity. Other sources involved in OISI, such as an increase in absorption due to increases in blood volume in capillaries or changes in light scattering due to microstructural changes in neural tissues, do not dominate at this wavelength.

The presentation of any visual stimulus induces increases of intrinsic signals over an area of several millimeters (global signals), but local signals that spread only for $\sim 0.5 \mathrm{~mm}$ are detected 
when the intrinsic signals for each stimulus condition is divided by the mean of intrinsic signals elicited by many stimuli (Wang et al., 1996, 1998) or by removing the global signals with spatial filtering (Tsunoda et al., 2001). The stimulus specific local signals (activity spots) are considered as supporting evidence for the existence of feature columns in IT cortex. Correlation between spiking activity of single cells and spot activity was examined in two ways. First, OISI with the critical stimulus features that were predetermined from spiking activity of single neurons revealed optical activity spots at the location where the single neurons were recorded (Wang et al., 1996, 1998). Second, selectivity of neural firing in activity spots was well correlated with selectivity of local optical signals at the spots (Tsunoda et al., 2001). Thus, spiking activity of neurons is well correlated in stimulus selectivity and in spatial localization with the local optical signals. It should be pointed out that, because intrinsic signals reflect population (spiking) activity of neurons, the best correlation between the local signals and neuronal firing is obtained at the level of MUA rather than single-unit activity (SUA).

Optical imaging with object images revealed that each object image is represented by the combined activity of multiple activity spots, and each spot represents a visual feature of the complex object image (Tsunoda et al., 2001). Finally, optical imaging with faces revealed that different viewing angles of a face were continuously and systematically mapped on the IT cortex (Wang et al., 1998). This might be part of a general tendency that similar visual features are mapped in nearby locations in IT cortex (Tanaka, 2003).

\section{Human and monkey fMRI}

Most fMRI studies in humans are based on an intrinsic signal, the BOLD contrast, which originates in a mismatch between the blood flow and oxygen extraction of the tissue. This mismatch leads to a net decrease in the concentration of deoxyhemoglobin in veins and capillaries around neuronal activity, which locally increases the magnetic resonance (MR) signal because deoxyhemoglobin acts as an MR contrast agent. In this respect, BOLD fMRI exhibits a striking contrast to OISI. OISI is sensitive to the early increase of deoxyhemoglobin in capillaries, whereas BOLD fMRI is more sensitive to the following oversupply of oxyhemoglobin (causing a decrease in the deoxyhemoglobin concentration) in capillaries and veins.

Even though in many human fMRI studies voxel sizes of $3 \mathrm{~mm}$ or larger are used, which are much larger than the size of the feature columns identified in monkey IT with invasive techniques, such studies have revealed surprisingly strong selectivity for comparisons between object classes such as faces versus objects and scenes (Kanwisher et al., 1997), (headless) bodies versus other object categories (Downing et al., 2001), and scenes/buildings versus objects and faces (Epstein and Kanwisher, 1998; Ishai et al., 1999). Similarly, in monkeys BOLD and contrast agentdependent cerebral blood volume (CBV) images acquired at a resolution of $1.25 \mathrm{~mm}$ revealed patches in monkey IT cortex that responded more strongly to faces or body parts than to objects (Tsao et al., 2003; Pinsk et al., 2005; Op de Beeck et al., 2008b). These findings are exciting, not the least because they were unexpected from previous electrophysiology experiments that had not shown large $(>1 \mathrm{~mm})$ cortical regions with a strong preference for specific object categories and with a consistent location across subjects.

Recent studies have used high-resolution fMRI (HR-fMRI) as the ultimate solution to better link fMRI to high-resolution op- tical imaging and electrophysiology data. Data suggest that regions that look like one large homogeneous blob $(\sim 1 \mathrm{~cm}$ diameter) at standard fMRI resolution can be distinguished into separate regions (Schwarzlose et al., 2005) or even more heterogenous clusters of voxels (Grill-Spector et al., 2006) at higher fMRI resolution. The difficulty of high-resolution fMRI, however, is that using smaller voxels decreases the signal-to-noise ratio (SNR), and increasing the resolution comes at the risk that (weak) activation may be missed. SNR improvement can be gained via parallel imaging, using high field scanners, or both. For parallel imaging, the sensitivity is improved by using multiple smaller radiofrequency coils (RF) coils. High magnetic fields increase the overall signal-to-noise, and the BOLD signal is also larger at high field. This makes the application of spin-echo (SE) sequences more practicable at high field (Goense et al., 2008). SE sequences have two advantages, compared with the commonly used gradient echo EPI (GE) sequence. SE sequences are less susceptible to signal loss from susceptibility artifacts from the air cavity that plague fMRI of the temporal lobe. Most importantly, SE sequences are thought to provide more localized activations from the capillary bed rather than from larger veins. For example, although ocular dominance columns and orientation columns in cat or human V1 are often difficult to discern with GE imaging, other fMRI methods (SE-BOLD imaging as well as cerebral blood volume/flow imaging) have higher specificity and allow for submillimeter resolution (Duong et al., 2003; Kim et al., 2004; Zhao et al., 2005; Yacoub et al., 2008). Nevertheless, it is yet unknown what is the maximal possible spatial resolution with HR-fMRI. This HR-fMRI limit depends on several factors: (1) signal-to-noise limitations, (2) the point spread function of the BOLD signal (which differs between SE and GE imaging), (3) the spatial scale of the neural clustering, and (4) the spatial scale of the separation between clusters having similar properties.

Many important functional questions remain unresolved given the currently available fMRI evidence. What factors underlie the large-scale selectivity to specific categories (e.g., faces and headless bodies) observed with fMRI? Is the selectivity stronger for these categories than for other categories? Is the spatial scale of representation (clustering of neurons with similar properties) larger and thus more easily measured with $\mathrm{fMRI}$ ? Does processing of these categories rely on different computations? Is category selectivity related to other characteristics of functional organization? How do these category-selective regions come about? fMRI studies have addressed some of these questions. First, it has been suggested that the strong selectivity for stimuli like faces might be related to their form or shape (Haxby et al., 2000), to specific processing related to faces (i.e., face recognition requires recognition at the exemplar level more than objects) (Gauthier, 2000), to semantic attributes in humans (Chao et al., 1999), and/or to eccentricity biases (Hasson et al., 2002) (regions that prefer faces also have a preference for foveal vs peripheral representations). Furthermore, selectivity in the ventral stream takes more than a decade to reach the adult-like state (Golarai et al., 2007). However, it is yet unclear how the strong selectivity for some object classes is related to these properties or their combination (Op de Beeck et al., 2008a). At the very least, a recent electrophysiological study suggests that the object category structure encoded by the firing rates of IT neurons corresponds to a level of visual form beyond a randomly selected set of moderately complex features (Kiani et al., 2007). 


\section{Correspondence between fMRI, optical imaging, and electrophysiology}

Previous work in other areas of the brain, most notably primary visual cortex, has focused on the relationship between fMRI, optical imaging, SUA and MUA, and LFPs. fMRI, single-neuron activity, and LFPs tend to correspond rather well in typical situations (Logothetis et al., 2001; Mukamel et al., 2005). This is possibly not that surprising, given that the input to a cluster of neurons is a significant determinant of their output. However, a few studies have been able to disentangle input and output of V1 neurons by manipulating the temporal characteristics of the stimuli. Synaptic activity and LFPs are less sensitive to adaptation (more sustained response to constant stimulation) and the frequency of flicker (nonbaseline response to rapidly flickering stimulus) compared with the spiking output of V1 neurons. In these cases, the fMRI signal seems to correlate more with LFPs than with the spiking output (Logothetis et al., 2001; Lauritzen, 2005; Viswanathan and Freeman, 2007). Dissociation between fMRI/LFP and spiking activity can also be induced with pharmacological manipulations that specifically affect the spiking output (Rauch et al., 2008). However, the correlation between spikes and LFP may depend on the degree of intercorrelations between the firing of neurons, and there is still some controversy about the exact relationship between these signals (Nir et al., 2008; Viswanathan and Freeman, 2008).

Here, we are mainly interested in the correspondence between these signals across spatial locations. A first point of consideration is the resolution and spatial spread of each technique. MUA and SUA sample the output of neurons, whereas LFP is a weighted signal dominated by dendritic input (Logothetis, 2003). MUA and SUA have highest spatial resolution, whereas LFPs are correlated across larger distances, often larger than feature columns or ocular dominance or orientation columns in V1. In V1, the spatial spread of LFP signals is of the order of a few millimeters (Juergens et al., 1999; Goense and Logothetis, 2008), whereas in IT the LFP signal spans a larger region, $\sim 5-8 \mathrm{~mm}$ (Kreiman et al., 2006). In IT, LFPs were correlated across sites with a much larger distance than multiunit activity, and in addition LFP selectivity could not be predicted very well from multiunit activity averaged across sites with varying diameter (Kreiman et al., 2006). This confirms that LFP represents also in IT a different signal than the MUA. Because the spatial extent of LFP is apparently larger than the spatial specificity of the local optical imaging signals, it is unclear how the specificity of the LFP relates to the specificity of the hemodynamic signals (optical imaging and fMRI).

Because optical imaging and fMRI both measure the hemodynamic response induced by neural activity, the two techniques should show similar results, as indeed they do in V1, in which correspondence was found between orientation columns measured by optical imaging and contrast agent-enhanced fMRI (Fukuda et al., 2006). This demonstration involved very highfield scanning $(9.4 \mathrm{~T})$ and contrast agent-enhanced CBVweighted fMRI in cats, as well as analysis techniques that allowed removal of potentially existing global nonselective signals. As mentioned above, GE-BOLD fMRI might lack sufficient spatial specificity to show a similar correspondence. Similar experiments are not straightforward in primate IT, because typically the effective fMRI resolution is lower $(\sim 2 \mathrm{~mm})$ and the spots seen in optical imaging are not larger than in cat V1. At current fMRI resolutions, we expect to get a weighted average of the spots/ feature columns within a voxel, which may fall apart as the resolution increases. The reported fMRI selectivity for unfamiliar shapes in monkey IT cortex (Op de Beeck et al., 2008b) might reflect such subsampling of very selective feature columns, but higher-resolution scanning is needed to test this hypothesis.

Many unknowns exist about the degree of correspondence between the invasive techniques and fMRI, but some correspondence is definitely present. For example, in monkeys it has been shown that fMRI-identified face-selective patches in the temporal lobe (six per hemisphere) contain a striking majority of neurons that are face-selective (Tsao et al., 2006). Functional connectivity experiments have already revealed the connectivity of the six face patches (Moeller et al., 2008), and electrophysiological experiments might pinpoint the unique step in face recognition performed by each of the different face patches.

However, many questions remain. First of all, faces may be a special category of stimuli, and we can question how these findings translate to other object categories. Furthermore, the properties of the fMRI-identified face patches have not been exhaustively compared with the properties of patches and columns as defined electrophysiologically through a dense sampling of single-unit activity both inside and outside the fMRI-identified face patches. Although the currently available evidence is very suggestive that face-selective patches have different properties than feature columns, a definitive answer awaits a better understanding of the functional and anatomical properties of both patches and columns. Finally, it is nontrivial to compare results across species. Homology of regions across species beyond early sensory areas is complex and often debatable (Kaas, 2008); the spatial scale of structures may differ across species (Adams et al., 2007). Furthermore, performing equivalent experiments across species is difficult even with fMRI. For example, should the comparison of face-selective responses across species use the same stimuli (e.g., human faces) or conspecific stimuli (human faces for humans and monkey faces for macaques)?

\section{Conclusion}

We are far from a complete understanding of the fine-scale and large-scale spatial organization of the cortical regions important for object and face recognition. Nevertheless, we have already experienced the power of a combined application of multiple neuroscientific techniques that measure functional organization at different spatial scales.

Ultimately, we apply these methods in the temporal lobe to understand how the brain recognizes and categorizes objects. The spatial organization of object representations is only one part of this general question, and it is the part for which the combined application of these neuroscientific techniques is most useful. However, neighboring single neurons often have clearly different selectivity properties and functions. Such differences are necessarily confounded, and underestimated, by techniques that pool signals over larger numbers of neurons, such as optical imaging and fMRI. Thus, the "gold standard" for understanding neural mechanisms remains single-unit electrophysiology. Nevertheless, the coarser-scale techniques can reveal large-scale patterns of organization that would otherwise go undetected, thereby helping single-unit electrophysiologists to target their electrodes better. In addition, the noninvasive techniques allow comparison between human and nonhuman brains, thereby providing a strong test of human-monkey correspondences and even allowing human studies to lead targeted electrophysiological experiments.

There are several outstanding questions that will only be solved by the combined application of all these methods, direct in the same study or indirect in different studies/laboratories. These 
questions center on the relationships between the different methods, the factors that underlie spatial organization in the temporal lobe at multiple scales, and how this multiple-scale organization emerges in the light of plasticity during development and adulthood. At a very basic level, we still do not know why we see clustering for particular functional properties (e.g., does the clustering of face cells in patches contribute to the speed and efficiency of face recognition?). Answering questions like this one will bring us closer to a full understanding of how the spatial organization of the temporal lobe relates to its functional role in visual object and face recognition.

\section{References}

Adams DL, Sincich LC, Horton JC (2007) Complete pattern of ocular dominance columns in human primary visual cortex. J Neurosci 27:10391-10403.

Baylis GC, Rolls ET, Leonard CM (1987) Functional subdivisions of the temporal lobe neocortex. J Neurosci 7:330-342.

Chao LL, Haxby JV, Martin A (1999) Attribute-based neural substrates in temporal cortex for perceiving and knowing about objects. Nat Neurosci 2:913-919.

Dean P (1976) Effects of inferotemporal lesions on the behavior of monkeys. Psychol Bull 83:41-71.

Desimone R, Gross CG (1979) Visual areas in the temporal cortex of the macaque. Brain Res 178:363-380.

Desimone R, Albright TD, Gross CG, Bruce C (1984) Stimulus-selective properties of inferior temporal neurons in the macaque. J Neurosci 4:2051-2062.

DiCarlo JJ, Cox DD (2007) Untangling invariant object recognition. Trends Cogn Sci 11:333-341.

DiCarlo JJ, Maunsell JH (2003) Anterior inferotemporal neurons of monkeys engaged in object recognition can be highly sensitive to object retinal position. J Neurophysiol 89:3264-3278.

Downing PE, Jiang Y, Shuman M, Kanwisher N (2001) A cortical area selective for visual processing of the human body. Science 293:2470-2473.

Duong TQ, Yacoub E, Adriany G, Hu X, Ugurbil K, Kim SG (2003) Microvascular BOLD contribution at 4 and $7 \mathrm{~T}$ in the human brain: gradientecho and spin-echo fMRI with suppression of blood effects. Magn Reson Med 49:1019-1027.

Epstein R, Kanwisher N (1998) A cortical representation of the local visual environment. Nature 392:598-601.

Fujita I, Tanaka K, Ito M, Cheng K (1992) Columns for visual features of objects in monkey inferotemporal cortex. Nature 360:343-346.

Fukuda M, Moon CH, Wang P, Kim SG (2006) Mapping iso-orientation columns by contrast agent-enhanced functional magnetic resonance imaging: reproducibility, specificity, and evaluation by optical imaging of intrinsic signal. J Neurosci 26:11821-11832.

Gauthier I I (2000) What constrains the organization of the ventral temporal cortex? Trends Cogn Sci 4:1-2.

Gochin PM, Miller EK, Gross CG, Gerstein GL (1991) Functional interactions among neurons in inferior temporal cortex of the awake macaque. Exp Brain Res 84:505-516.

Goense JB, Logothetis NK (2008) Neurophysiology of the BOLD fMRI signal in awake monkeys. Curr Biol 18:631-640.

Goense JB, Zappe AC, Logothetis NK (2007) High-resolution fMRI of macaque V1. Magn Reson Imaging 25:740-747.

Goense JB, Ku SP, Merkle H, Tolias AS, Logothetis NK (2008) fMRI of the temporal lobe of the awake monkey at 7 T. Neuroimage 39:1081-1093.

Golarai G, Ghahremani DG, Whitfield-Gabrieli S, Reiss A, Eberhardt JL, Gabrieli JD, Grill-Spector K (2007) Differential development of highlevel visual cortex correlates with category-specific recognition memory. Nat Neurosci 10:512-522.

Grill-Spector K, Sayres R, Ress D (2006) High-resolution imaging reveals highly selective nonface clusters in the fusiform face area. Nat Neurosci 9:1177-1185.

Grinvald A, Lieke E, Frostig RD, Gilbert CD, Wiesel TN (1986) Functional architecture of cortex revealed by optical imaging of intrinsic signals. Nature 324:361-364

Gross CG, Bender DB, Rocha-Miranda CE (1969) Visual receptive fields of neurons in inferotemporal cortex of the monkey. Science 166:1303-1306.
Gross CG, Rocha-Miranda CE, Bender DB (1972) Visual properties of neurons in inferotemporal cortex of the macaque. J Neurophysiol 35:96-111.

Harel N, Lin J, Moeller S, Ugurbil K, Yacoub E (2006) Combined imaginghistological study of cortical laminar specificity of fMRI signals. Neuroimage 29:879-887.

Hasson U, Levy I, Behrmann M, Hendler T, Malach R (2002) Eccentricity bias as an organizing principle for human high-order object areas. Neuron 34:479-490.

Haxby JV, Ishai II, Chao LL, Ungerleider LG, Martin I I (2000) Object-form topology in the ventral temporal lobe. Response to I. Gauthier (2000). Trends Cogn Sci 4:3-4.

Hung CP, Kreiman G, Poggio T, DiCarlo JJ (2005) Fast readout of object identity from macaque inferior temporal cortex. Science 310:863-866.

Ishai A, Ungerleider LG, Martin A, Schouten JL, Haxby JV (1999) Distributed representation of objects in the human ventral visual pathway. Proc Natl Acad Sci U S A 96:9379-9384.

Ito M, Tamura H, Fujita I, Tanaka K (1995) Size and position invariance of neuronal responses in monkey inferotemporal cortex. J Neurophysiol $73: 218-226$.

Juergens E, Guettler A, Eckhorn R (1999) Visual stimulation elicits locked and induced gamma oscillations in monkey intracortical- and EEGpotentials, but not in human EEG. Exp Brain Res 129:247-259.

Kaas JH (2008) The evolution of the complex sensory and motor systems of the human brain. Brain Res Bull 75:384-390.

Kanwisher N, McDermott J, Chun MM (1997) The fusiform face area: a module in human extrastriate cortex specialized for face perception. J Neurosci 17:4302-4311.

Kiani R, Esteky H, Mirpour K, Tanaka K (2007) Object category structure in response patterns of neuronal population in monkey inferior temporal cortex. J Neurophysiol 97:4296-4309.

Kim DS, Ronen I, Olman C, Kim SG, Ugurbil K, Toth LJ (2004) Spatial relationship between neuronal activity and BOLD functional MRI. Neuroimage 21:876-885.

Kobatake E, Tanaka K (1994) Neuronal selectivities to complex object features in the ventral visual pathway of the macaque cerebral cortex. J Neurophysiol 71:856-867.

Kreiman G, Hung CP, Kraskov A, Quiroga RQ, Poggio T, DiCarlo JJ (2006) Object selectivity of local field potentials and spikes in the macaque inferior temporal cortex. Neuron 49:433-445.

Lauritzen M (2005) Reading vascular changes in brain imaging: is dendritic calcium the key? Nat Rev Neurosci 6:77-85.

Logothetis NK (2003) The underpinnings of the BOLD functional magnetic resonance imaging signal. J Neurosci 23:3963-3971.

Logothetis NK, Pauls J, Augath M, Trinath T, Oeltermann A (2001) Neurophysiological investigation of the basis of the fMRI signal. Nature 412:150-157.

Moeller S, Freiwald WA, Tsao DY (2008) Patches with links: a unified system for processing faces in the macaque temporal lobe. Science 320:1355-1359.

Mukamel R, Gelbard H, Arieli A, Hasson U, Fried I, Malach R (2005) Coupling between neuronal firing, field potentials, and FMRI in human auditory cortex. Science 309:951-954.

Nir Y, Dinstein I, Malach R, Heeger DJ (2008) BOLD and spiking activity. Nat Neurosci 11:523-524; author reply 524.

Op de Beeck H, Vogels R (2000) Spatial sensitivity of macaque inferior temporal neurons. J Comp Neurol 426:505-518.

Op de Beeck HP, Haushofer J, Kanwisher NG (2008a) Interpreting fMRI data: maps, modules and dimensions. Nat Rev Neurosci 9:123-135.

Op de Beeck HP, Deutsch JA, Vanduffel W, Kanwisher NG, DiCarlo JJ (2008b) A stable topography of selectivity for unfamiliar shape classes in monkey inferior temporal cortex. Cereb Cortex 18:1676-1694.

Perrett DI, Rolls ET, Caan W (1982) Visual neurones responsive to faces in the monkey temporal cortex. Exp Brain Res 47:329-342.

Pinsk MA, DeSimone K, Moore T, Gross CG, Kastner S (2005) Representations of faces and body parts in macaque temporal cortex: a functional MRI study. Proc Natl Acad Sci U S A 102:6996-7001.

Rauch A, Rainer G, Logothetis NK (2008) The effect of a serotonin-induced dissociation between spiking and perisynaptic activity on BOLD functional MRI. Proc Natl Acad Sci U S A 105:6759-6764.

Saleem KS, Tanaka K, Rockland KS (1993) Specific and columnar projection from area TEO to TE in the macaque inferotemporal cortex. Cereb Cortex 3:454-464. 
Schwarzlose RF, Baker CI, Kanwisher N (2005) Separate face and body selectivity on the fusiform gyrus. J Neurosci 25:11055-11059.

Tanaka K (2003) Columns for complex visual object features in the inferotemporal cortex: clustering of cells with similar but slightly different stimulus selectivities. Cereb Cortex 13:90-99.

Tanigawa H, Wang Q, Fujita I (2005) Organization of horizontal axons in the inferior temporal cortex and primary visual cortex of the macaque monkey. Cereb Cortex 15:1887-1899.

Tsao DY, Freiwald WA, Knutsen TA, Mandeville JB, Tootell RB (2003) Faces and objects in macaque cerebral cortex. Nat Neurosci 6:989-995.

Tsao DY, Freiwald WA, Tootell RB, Livingstone MS (2006) A cortical region consisting entirely of face-selective cells. Science 311:670-674.

Tsunoda K, Yamane Y, Nishizaki M, Tanifuji M (2001) Complex objects are represented in macaque inferotemporal cortex by the combination of feature columns. Nat Neurosci 4:832-838.

Viswanathan A, Freeman RD (2007) Neurometabolic coupling in cerebral cortex reflects synaptic more than spiking activity. Nat Neurosci 10:1308-1312.

Viswanathan A, Freeman RD (2008) Reply to "BOLD and spiking activity." Nat Neurosci 11:524.

Wallis G, Rolls ET (1997) Invariant face and object recognition in the visual system. Prog Neurobiol 51:167-194.

Wang G, Tanaka K, Tanifuji M (1996) Optical imaging of functional organization in the monkey inferotemporal cortex. Science 272:1665-1668.

Wang G, Tanifuji M, Tanaka K (1998) Functional architecture in monkey inferotemporal cortex revealed by in vivo optical imaging. Neurosci Res 32:33-46.

Yacoub E, Harel N, Ugurbil K (2008) High-field fMRI unveils orientation columns in humans. Proc Natl Acad Sci U S A 105:10607-10612.

Zhao F, Wang P, Hendrich K, Kim SG (2005) Spatial specificity of cerebral blood volume-weighted fMRI responses at columnar resolution. Neuroimage 27:416-424. 